\title{
Fatal infection with Mycobacterium fortuitum associated with oesophageal achalasia
}

\author{
G VARGHESE, R SHEPHERD, P WATT, J H BRUCE \\ From the Departments of Medicine and Pathology, The Queen's University of Belfast
}

Association of chronic pulmonary disease and oesophageal disorders is well recognised. Fatal infections are, however, relatively infrequent. Two previously reported fatal cases have been due to pulmonary infection with Mycobacterium fortuitum, one diagnosed at necropsy in a woman with polyarthritis and achalasia previously treated with corticosteroids ${ }^{1}$ and the second associated with cystic fibrosis, ${ }^{2}$ the duration of illness having been eight months after the isolation of the organism. The present case is unusual in that the infection occurred in an otherwise healthy woman with asymptomatic achalasia and, unlike previous cases, the illness was acute and rapidly progressive.

\section{Case report}

A 29 year old school teacher presented to the hospital with a history of fever, sweating, and left sided chest pain of one week's duration. Eleven weeks previously she had had a normal delivery; she had suffered vomiting and loss of weight during her two pregnancies, but had had few symptoms after delivery. On admission she appeared ill, with a temperature of $39^{\circ} \mathrm{C}$. Impaired vocal resonance and bronchial breathing were noted over the left base. The haemoglobin concentration was $10.4 \mathrm{~g} / \mathrm{dl}$ and the white cell count $10 \times 10^{9} / 1$. The chest radiograph showed consolidation over the left base and a small area of consolidation over the right upper zone (fig 1), and the oesophagus was noted to be grossly dilated. She was treated as for aspiration pneumonia with penicillin, flucloxacillin, and metronidazole. Sputum examination initially showed no organisms, but subsequently showed acid fast bacilli. Antituberculous treatment was started with rifampicin, isoniazid, and ethambutol. She remained febrile and showed little response to treatment; the chest radiograph two weeks after admission showed the areas of consolidation to be much more extensive (fig 2). She was transferred to the chest unit. She was febrile and acutely dyspnoeic. The haemoglobin concentration had fallen to $7.9 \mathrm{~g} / \mathrm{dl}$ and the arterial oxygen tension $\left(\mathrm{PaO}_{2}\right)$ was $7.8 \mathrm{kPa}$. She was transfused with three units of whole blood. Feeding was continued via a nasogastric tube. She received erythromycin in addition to the antituberculous drugs. Sputum culture subsequently yielded a rapidly growing mycobacterium identified as $M$ fortuitum. Amikacin was added to the treatment. Her general condition steadily worsened, with a fall in $\mathrm{PaO}_{2}$. She was moved to the intensive care unit and ventilated. Her tracheal

Address for reprint requests: Dr Grace Varghese, Belfast City Hospital, Belfast BT9 7AB.

Accepted 1 July 1987 aspirate repeatedly yielded $M$ fortuitum in culture. In vitro sensitivity studies showed that the organisms were resistant to all antibiotics except amikacin. She developed terminal coagulopathy and died four weeks after the initial presentation.

The oesophagus showed dilatation over the entire length except for the terminal $4 \mathrm{~cm}$. Both lungs showed extensive areas of consolidation. In several areas the lung tissue had broken down, resulting in abscess formation. Histological examination of the lung showed widespread evidence of aspiration of lipid material. The lung parenchyma showed heayily inflamed fibrous tissue with many giant cells, fibroblasts, and chronic inflammatory cells. No granulomas were identified. Ziehl-Neelsen staining showed numerous acid fast bacilli.

\section{Discussion}

The Mycobacterium fortuitum group of organisms (Runyou group IV) are characterised by their rapid growth at temperatures ranging from $25^{\circ}$ to $42^{\circ} \mathrm{C}$. These organisms were previously thought to be non-pathogenic and had been isolated from the sputum in patients with no clinical or radiological progression of pulmonary disease. ${ }^{3}$ The pathogenicity of the organisms has become more evident in recent years.

Most human infections are acquired by inoculation after

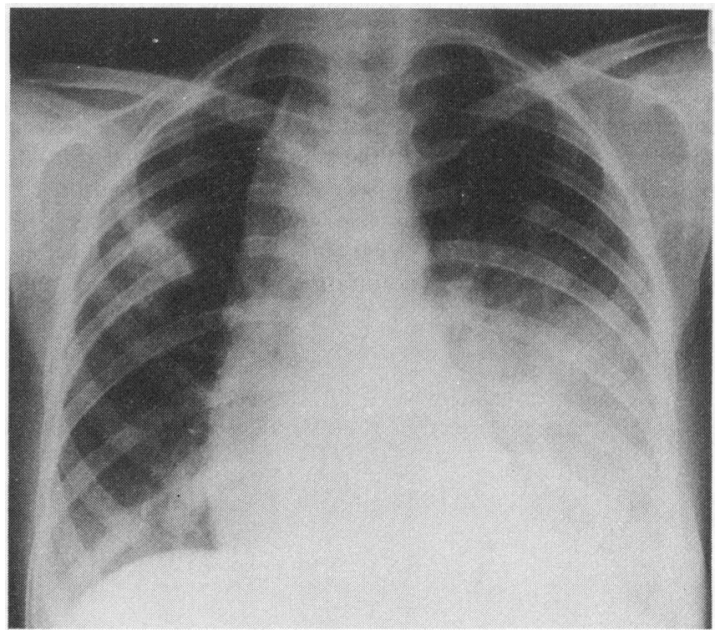

Fig 1 Initial chest radiograph showing the dilated oesophagus and consolidation of both lungs. 


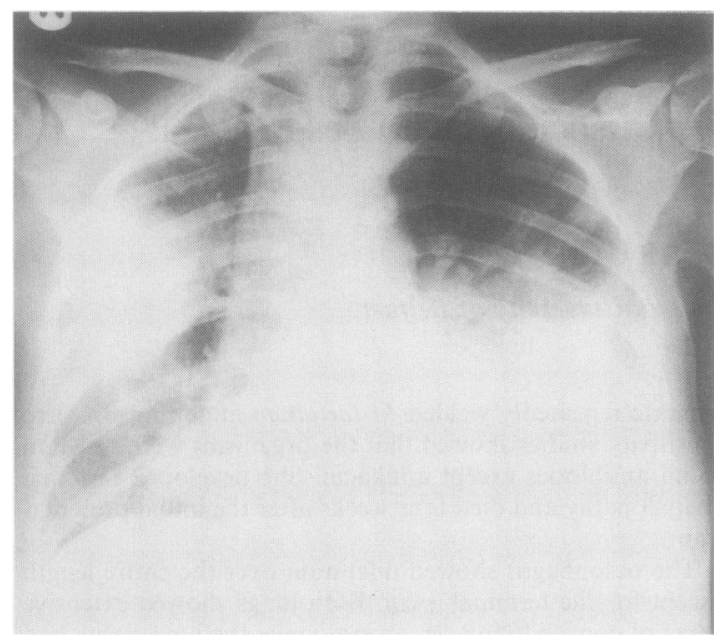

Fig 2 Radiograph two weeks later showing the considerable progression of the changes.

accidental trauma, surgery, or infection. Widely varying clinical syndromes may be encountered, the most common being infections of the skin and soft tissue. Outbreaks of wound infection have occurred after cardiothoracic surgery, renal dialysis, mammoplasty, and arthroplasty. ${ }^{45}$

Bronchopulmonary infections often occur after aspiration. Infections are most common in the presence of severe underlying lung disease, as in cystic fibrosis, and in those receiving immunosuppressive treatment.

An increased incidence of chronic pulmonary infection in achalasia and other oesophageal disorders has been found in previous surveys. ${ }^{6}$ The importance of fat and oily substances for the saprophytic mycobacterium has been emphasised in relation to the morbid effect. Laporte in his animal experiments showed that injection of fatty material with a saprophytic mycobacterium can produce intense lesions resulting in death. ${ }^{7}$ It is thought that the survival of the organisms surrounded by fat may be partly explained by their physical protection against enzymes and the favourable effect of a fatty environment on the growth of mycobacteria. The increased susceptibility to pulmonary disease in achalasia must therefore be related to the stagnation of nutrient fluids in the oesophagus, which provide an ideal culture medium for the growth of organisms that are otherwise non-pathogenic. ${ }^{8}$

The resistance of the organisms to standard antituberculous drugs and to most common antibiotics causes difficult problems in management. Some have shown susceptibility to amikacin, erythromycin, ethionamide, and doxycycline.

Even though the organism showed in vitro sensitivity to amikacin our patient had fulminating disease and failed to respond to standard antituberculous drugs and to the combination of amikacin and erythromycin. ${ }^{9}$

The acute onset and rapid progression of illness in this case seem to be unusual, yet the repeated isolation of $\boldsymbol{M}$ fortuitum from sputum and tracheal aspirates in the absence of any other organisms and the presence of numerous acid fast bacilli demonstrated at necropsy leave little doubt that the fatal infection was caused by $\boldsymbol{M}$ fortuitum.

We may speculate on whether the fatty environment in the oesophagus caused by stagnation of milk and other nutrient fluids may have altered the pathogenic behaviour of mycobacteria that were otherwise potentially non-pathogenic.

We wish to thank the staff of the intensive care unit and the Department of Microbiology at the Royal Victoria Hospital for their help and guidance.

\section{References}

1 Baneriee R, Hall R, Hughes GRV. Pulmonary Myobacterium fortuitum infection in association with achalasia of the oesophagus. Br J Dis Chest 1970;64:112-7.

2 Efthimiou J, Smith MJ, Hodson ME, Batten JC. Fatal pulmonary infection with Mycobacterium fortuitum in cystic fibrosis. $\mathrm{Br}$ Dis Chest 1984;78:299-302.

3 Awe RJ, Gangadharam PR, Jenkins DE. Clinical significance of Mycobacterium fortuitum in pulmonary disease. Am Rev Respir Dis 1973;108:1230-4.

4 WE Sanders Jr. Other mycobacterium species. In: Mandell GL, Douglas RG, Bennett JE, eds. Principles and practice of infectious disease. New York: John Wiley and Sons, 1985 1413-23.

5 Robiesek F, Daugherty HK, Cook JW, et al. Mycobacterium fortuitum epidemics after open heart surgery. $J$ Thorac Cardiovasc Surg 1978;75:91-5.

6 Vivas JR, Palmer ED. Incidence of chronic pulmonary disease among patients with esophageal disorders. Dis Ches 1957;31:568-73.

7 Laporte R. A l'étude des bacilles paratuberculeux. Ann Inst Pasteur 1940;65:282-325.

8 Gibson JB. Infection of the lungs by saprophytic mycobacteria in achalasia of the cardia with a report of a fatal case showing lipoid pneumonia due to milk. J Pathol Bacteriol 1953;651 239-51.

9 Dalovisio JR, Pankey GA. Problem in diagnosis and therapy of Mycobacterium fortuitum infections. Am Rev Respir Dis 1978;117:625-30. 